\title{
ESTUDOS DE BRIÓFITAS DO BRASIL: 6. PHYLLOGONIACEAE (BRYOPSIDA) (1)
}

\author{
Olga Yano (2) \\ Zélia Rodrigues de Mello (3)
}

RESUMO - O estudo da familia monotípica Phyllogoniaceae (Bryopsida) baseiase na descrição da espécie Phyllogonium viride Brid. e inclui apresentação de esquemas e desenhos necessários para identificação sistemática e sua distribuição geográfica no Brasil.

Palavras-chave: Phyllogonium viride, musgo. distribuição, Brasil.

ABSTRACT - A studyu of the monotypic family Phyllogoniaceae (Bryopsida) is presented, including for the species Phyllogonium viride Brid. a morphological description, illustrations and its distribution in Brazil.

Key words: Phyllogonium viride, Musci, distribution, Brazil.

\section{Introdução}

A família Phyllogoniaceae, na revisão taxonômica de Lin (1983), é considerada monogenérica, isto é, possui apenas o gênero Phyllogonium Brid. Isto porque os outros gêneros Horikawaea Nog., Orthorrhynchidium Ren. \& Card. e Pursellia Lin, são membros de Pterobryaceae (Lin, 1984) e Catagonium C. Muell. ex Broth. é considerado gênero-tipo da nova família Catagoniaceae proposta por Buck \& Ireland (1985).

1. O título da série "Contribuição ao inventário dos Musci brasiieiros" foi substituído por "Estudos de Briófitas no Brasil".

2. Instituto de Botânica, Caixa Postal 4005, 01051 São Paulo, SP, Brasil.

3. Universidade Santa Cecília dos Bandeirantes, Caixa Postal 1213, 11045 Santos, SP, Brasil. 
No Brasil, Phyllogoniaceae está representada por uma única espécie Phyllogonium viride Brid. Apesar do catálogo de musgos brasileiros apresentado por Yano (1981a) ter relatado mais quatro espécies, $P$. caldense C. Muell., $P$. fulgens (Hedw.) Brid., P. immersum Mitt. e P. riograndense C. Muell. ex Broth., todas estas espécies são consideradas sinônimos de $P$. viride por Lin (1983).

O objetivo deste trabalho é contribuir para o inventário da flora briotífica, cuja série foi iniciada com a publicação de Helicophyllaceae (Yano, 1979; 1984a), Phyllodrepaniaceae (Yano, 1981b), Aytoniaceae (Yano, 1981c), Racopilaceae (Yano, 1984b), Rhachitheciaceae (Yano, 1985), Rhizogoniaceae (Yano, 1986) e também ampliar a distribuição geográfica de cada espécie estudada.

\section{Material e Métodos}

A metodologia de coleta e preservação de material foi baseada em Yano (1984c). Os espécimes examinados pertencem, em sua maioria, aos herbários Maria Eneyda P. Kauffmann Fidalgo (SP) e Jardim Botânico do Rio de Janeiro (RB). Foram também examinados materiais dos herbários Barbosa Rodrigues, de Itajaí (HBR) e Herbarium Anchieta, do Instituto Anchietano de Pesquisas, de São Leopoldo (ASSL).

O material coletado para este trabalho possui duplicatas para eventual intercâmbio.

\section{Resultados}

Phyllogonium viride Brid., Bryol. Univ. 2: 273. 1827 (Fig. 1-14). Localidadetipo: Brasil (B).

Sinonímias:

$=P$. immersum Mitt., J. Linn. Soc. Bot. 12: 423.1869.

Localidade-tipo: Brasil, Paraná (BM).

$=P$. caldense C. Muell., J. Mus. Godeffroy 3(6): 69, 1874.

Localidade-tipo: Brasil, Minas Gerais (não examinado).

$=P$. riograndense C. Muell. ex. Broth., Bih. K. svenska VetensAkad. Handl. 3(7): 39. 1900.

Localidade-tipo: Brasil, Rio Grande do Sul (BM).

Gametófitos longamente pêndulos, até $500 \mathrm{~mm}$ de comprimento, verdeamarelados, castanho-dourados, lustrosos. Filídios dos ramos pendentes dispos- 
tos disticamente, 3-4mm de comprimento, lanceolados e oblongos, cimbiformes ou naviculares terminando em rostro nitidamente recurvado, nunca ondulado; margens inteiras; costa curta e dupla ou ausente; as células alares constituindo um grupo bem delimitado de células avermelhadas e espessadas, parenquimatosas indistintas, as demais estreitas alongadas a lineares, com paredes tipicamente estranguladas (porosas). Filídios periqueciais com o dobro do comprimento dos ramos, $1-8 \mathrm{~mm}$ de comprimento, largamente ovalados, longamente acuminados, fortemente côncavos, margens inteiras, às vezes serreadas a espinulosas. Filídios dos ramos primários pequenos e côncavos, não dispostos disticamente, $1 \mathrm{~mm}$ de comprimento. Dióicos. Esporófito imerso; seta muito curta, 0,3mm de comprimento; cápsula globosa ou largamente oblonga; opérculo cônico-rostrado; calíptra pequena, cuculada ou mitriforme de base inteira, densamente pilosa; peristômios simples, dentes, $\pm 250 \mu \mathrm{m}$ de comprimento, hialinos, irregularmente fendidos; esporos de tamanho variado, $25-42 \mu \mathrm{m}$, esféricos a subesféricos e finamente ornamentados. Anterídios em forma de botão minúsculo nas axilas de filídios dos ramos.

Distribuição geográfica - Brasil, Minas Gerais, Rio de Janeiro, São Paulo, Paraná, Santa Catarina e Rio Grande do Sul (Yano, 1981a, como P. immersum e Lin, 1983); Pernambuco (Yano \& Andrade-Lima, 1987, como P. immersum) e Bahia (Lin, 1983).

Espécimes examinados - Pernambuco, município de Taquaritinga do Norte, sobre tronco vivo na mata ao lado da torre de microondas, col. O. Yano \& D. Andrade-Lima 2634, 24-VIII-1980 (SP 170670); idem, Morro Cafundó, sobre tronco de Inga sp., na mata da Serra Cafundó, col. O. Yano \& D. Andrade-Lima 2601, 24-VIII-1980 (SP 170669); município de Caruaru, Fazenda Caruaru, sobre galhos de árvores na mata únida do Brejo dos Cavalos, col. O. Yano \& D. Andrade-Lima 2770, 29-VIII-1980 (SP 170671); município de São Caetano, Serra Pelada, sobre tronco de munguba, na Serra Pelada perto da torre de microondas, col. O. Yano \& D. Andrade-Lima 2815, 29-VIII-1980 (SP 170674); Brejo da Madre de Deus, propriedade Bituri Grande, Mata de Pontaria, col. D. Andrade-Lima 81-6686, 20-II-1981 (SP 170715). Alagoas, União dos Palmares, Engenho Santo Antônio, dentro da mata, próximo ao rio, col. I. Pontual 65-86, 2-III-1965 (IPA 14102); município de Boca da Mata, alto da Serra de Naceia, epífita, col. D. Andrade-Lima 80-6657, 29-X-1980 (SP 170707). Bahia, Jacobina, Serra do Tombador, col. I. Pontual, 5-VIII-1980 (SP 149679). Minas Gerais, município de Santa Bárbara, Serra do Areião, col. F. C. Hoehne s/n, 23-I-1920, det. T. Herzog, como P. immersum (SP 88934); idem, Água limpa, Serra do Gongo, col. F. C. Hoehne s/n, -I-1921, det. T. Herzog como P. Immersum (SP 88933); Santa Bárbara, col. F. C. Hoehne, -I-1921, det. T. Herzog, como P. immersum (SP 5563); município de Camanducaia, Monte Verde, sobre tronco de árvore, mata úmida e sombria, col. Dayse V. Martins \& C. L. Sant'anna, 21-II-1979 (SP 147561); município de Santa Bárbara, Parque Natural do Caraça, picada para a gruta de Lurdes. sobre tronco vivo, mata úmida, col. O. Yano \& J. R. Pirani 7347, 27-V-1983 (SP 
182413); idem, col. O. Yano \& J. R. Pirani 7377, 28-V-1983 (SP 182442); município de Caparaó Novo, Parque Nacional de Caparaó, along the road from park entrana to end of road (Tronqueira), ca. $1000-1970 \mathrm{~m}, 20^{\circ} 27^{\prime} \mathrm{S}, 41^{\circ} 50^{\prime} \mathrm{W}$, dryish low forest, col. D. W. Vital \& W. R. Buck 11557, 15-IX-1984, det. D. Vital como $P$. fulgens (SP 202038); idem, at Vale Verde, small water fall and adjacent humid forest, ca. $1000 \mathrm{~m}$, ca. $20^{\circ} 27^{\prime} \mathrm{S}, 41^{\circ} 50^{\prime} \mathrm{W}$, col. D. M. Vital \& W. R. Buck 11655, 15-IX-1984, det. D. Vital como P. immersum (SP 202116); idem, epífita de árvore viva, próximo ao curso de água, pendente na maioria das árvores, col. Fernando \& Dulce 188, 30-IV-1988 (CESJ 22515). Espírito Santo, município de Santa Tereza, Rio Timbuí, sobre pedras junto do Rio Timbuí, col O. Yano 3633, 5-VII-1981 (SP 170934); idem, perto da Reserva Biológica de Nova Lombardia, no barranco de solo arenoso, na estrada, col O. Yano, D. P. Santos \& J. R. Pirani 4923, 23-XI-1982 (SP 172434); município de Serra, Estação Biológica de Mestre Álvaro, sobre pau podre perto da cachoeira, col. O. Yano, D. P. Santos \& J. R. Pirani 4778, 21-XI-1982 (SP 172370 ); idem, sobre tronco vivo, topo da serra, $\pm 700 \mathrm{~m}$ de altitude, col. O. Yano, D. P. Santos \& J. R. Pirani 4828, 21-XI-1982 (SP 172390); município de Muniz Freire no Km 138 da BR-262, no barranco argiloso perto de Polytrichum, ao lado da estrada, col. O. Yano, D. P. Santos \& J. R. Pirani 4959, 24 XI-1982 (SP 172460). Rio de Janeiro, col. Ule, - 1875 (RB 210571, ex Herb. Schwacke 904); col. ? (RB 93420, ex Herb. Damazio 10432); município de Resende, Parque Nacional do Itatiaia, Maromba, sobre galhos de um arbusto, entre as pedras, nas bordas do ribeirão, col. D. M. Vital 790, 791, 1-IV-1966 (SP 89410, SP 89411); idem, sobre tronco fino, na estreita faixa de mata, à beira do ribeirão $\pm 6 \mathrm{~m}$ da margem do Ribeirão, col. D. M. Vital 801, 802, 1-IV-1966 (SP 89416, SP 89417); idem, south face of Mt. Itatiaia, in valley of the creek Rio Campo Belo, at Maromba, along path to waterfall, alt. $1150 \mathrm{~m}$ well developed secondary forest, growing on more lighted side of the trunk, col. G. Eiten \& L. T. Eiten 6410, 31-X-1965 (SP 127730); idem, near Lago Azul, alt. 820m, col. G. Eiten \& L. T. Eiten 7275, 23-VII-1966 (SP 90329); idem, Rio Campo Belo, lote 17 and adjacent areas (ca. $22^{\circ} 30^{\prime} \mathrm{S}, 44^{\circ} 35^{\prime} \mathrm{W}$ ) evergreen tropical forest, Myrtaceae common, elev. ca. 1000m, on log, col. L. R. LANDRUM 2184, 17-X-1977 (RB 210555); idem, picada para Três Picos, sobre tronco vivo junto da picada, col. O. Yano \& D. P. Santos 7588, 21-VI-1983 (SP 182644); idem, Abrigo 1, $\pm 800 \mathrm{~m}$ alt., sobre tronco vivo, col. O. Yano \& S. R. Visnardi 9771, 30-XI-1985 (SP 206474); idem, Lago Azul, sobre tronco vivo perto do lago, col. O. Yano \& S. R. Visnardi 9827, 30-XI-1985 (SP 206529); Mauá, Iatatiaia, na mata pendente das árvores, col. M. C. Vaugham Bandeira 411, 8-II-1923, det. Brotherus (RB 210604); Rio de Janeiro, Corcovado, aqueduto das Paineiras, pendente de rochas, col. M. C. Vaugham Bandeira 410, 17-III-1925, det. Brotherus (RB 210606); Teresópolis, Poço d'antas, em árvore viva na mata em lugar de alguma sombra, col. M. C. Vaugham Bandeira 512, 23-III-1926 (RB 210605); Rio de Janeiro, Pedra de Gávea, $\pm 800 \mathrm{~m}$, saxícola, umbrófila, às vezes epífita, col. D. Sucre 1628, 5-X-1967 (RB 136879, como Estado da Guanabara); idem, Parque Nacional da Tijuca, Bico de Papagaio, and areas near by (ca. 
$22^{\circ} 50^{\prime} \mathrm{S}, 43^{\circ} 20^{\prime} \mathrm{W}$ ), evergreen tropical forest, elevation $800-1000 \mathrm{~m}$, on rock, col. L. R. Landrum 2213, 24-X-1977 (RB 210550). Sāo Paulo, col. F. C. Hoehne 435, s/d (SP 33060, ex herb. Comissão Geográfica e Geológica de S. Paulo); São Paulo, Bosque da Saúde, epífita, col. F. C. Hoehne, 29-VIII-1920, det. T. Herzog como $P$. immersum (SP 88932); idem, estrada do Vergueiro, col. F. C. Hoehne, XII-1920, det. T. Herzog como P. immersum (SP 5562); idem, serra da Cantareira, col. B. Pickel 4395, 31-VII-1939 (IPA 8757, como $P$. immersum Mitt.); idem, Cantareira, sobre árvore viva na mata, col. A. Sehnem, 20-CII-1960 (ASSL 76976); município de Santo André, Alto da Serra, epífita, col. F. C. Hoehne s/n, 21-IV-1920, det. T. Herzog como $P$. immersum (SP 4337, SP 88936); idem, Estação Biológica, col. A. B. Joly 1033, 3IV-1951, det. D. M. Vital como P. fulgens (SP 90372); idem, Biological Station on of Alto da Serra, $1 / 2-1 \mathrm{Km}$ west of village of Paranapiacaba, large-leaved moss, epiphytic on leaves of an epiphytic orchid, in scrubby forest, col. G. Eiten \& L. T. Eiten 5038, 28-X-1962 (SP 90327); idem, moss on roots of an epiphytic Peperomia in scrubby forest, col. G. Eiten \& L. T. Eiten 5030, 28-X-1962 (SP 90328); Campos do Jordão, sobre Podocarpus, col. F. C. Hoehne s/n, 17-IX-1923, det. T. Herzog como P. immersum (SP 88935); idem, sobre tronco e galho, na mata secundária (no limite do campo) $\pm 1,5 \mathrm{Km}$ a NNW do pico de Itapeva, \pm 1900 m alt., col. D. M. Vital 694, 4-III-1966 (SP 89370); idem, Parque Estadual São José dos Alpes, 1900m, pendentes de árvores, mata nebulosa, col. M. P. Bautista \& G. Barroso 237, 22-XI-1975 (RB 182038); idem, sobre galhos e troncos finos em uma pequena área e mata entre o Horto e a Represa Nova, $\pm 22^{\circ} 38^{\prime} \mathrm{S}, 45^{\circ} 33^{\prime} \mathrm{W}$, col. D. M. Vital 9628 , 22XI-1980, det. D. M. Vital como P. fulgens (SP 149787); idem, sobre tronco e galhos finos de arbusto, ao longo de um riacho, próximo à represa nova, \pm $23^{\circ} 43^{\prime}$ S, $45^{\circ} 39$ 'W, col. D. M. Vital 9644, 22-XI-1980, det. D. M. Vital como $P$. fulgens (SP 149795); idem, sobre tronco fino, na mata úmida e brejosa, ca. $3 \mathrm{Km}$ W da engarrafadora Minalba, $\pm 23^{\circ} 43^{\prime} \mathrm{S}, 45^{\circ} 39^{\prime} \mathrm{W}$, col. D. M. Vital 9702, 22-XI-1980 (SP 149817); idem, Fazenda Lagoinha, 1600m, col. W. Bokermann s/n, 10-I-1981 (SP 170459); Ribeirão Pires, stat. viae ferreae inter São Paulo e Santos, in superfície corticis arborum, locis umbrosis, col. A. Baranov 22, 21-VI-1959 (SP 146127); município de Mogi das Cruzes (near border with munic. de Salesópolis = Biological Station of Boracéia, $23^{\circ} 38^{\prime}-39^{\prime} \mathrm{S}, 45^{\circ} 54^{\prime} \mathrm{W}$, alt. 800m, col. E. Eiten \& L. T. Eiten 2512, 26-I-1961 (SP 89918); Pindamonhangaba, Pico de Itapeva, sobre tronco de árvore, na mata secundária, $\pm 3 \mathrm{Km}$ SSW do Pico, $\pm 1800 \mathrm{~m}$ alt., col. D. M. Vital 634, 25-I-1966 (SP 89342); idem, ca. $2 \mathrm{~m}$ do solo na mata úmida junto à cachoeira, $\pm 2 \mathrm{Km} \mathrm{NE}$ do Pico de Itapeva, col. D. M. Vital 1927, 14-XII-1971 (SP 90225); município de Cunha, Reserva Florestal de Bocaina, $\pm 1600 \mathrm{~m}$, espífita, umbrófila crescendo em mata úmida,col. D. Sucre 2886, D. J. Guimarães 80 \& P. I. S. Braga, 5-V-1968 (RB 139858); idem, epiphytiseg im Berg regenward 1500m, col. J. P. Frahm 1603, 21-VII-1977 (SP 147047); São Paulo, Parque Estadual das fontes do Ipiranga, growing from base to $5 \mathrm{~m}$ up on a living tree trunk in a humid secondary forest, col. O. Yano 238, 7-V-1975 (SP 122124); município de Juquitiba, BR-116, Km 
26 à direita (SP-Curitiba), sobre tronco árvore viva, $1 \mathrm{~m}$ do solo, col. O. Yano 556, 17-X-1976 (SP 136656); município de São Miguel Arcanjo, Reserva Florestal de Carlos Botelho, on branches of tree, along a stream, col. D. M. Vital 7130, 22-V-1977 (SP 135968); município de Silveira, on bark of a tree, in a semi-virgin forest, on sloping region of Serra da Bocaina $\left(22^{\circ} 43^{\prime} \mathrm{S}, 44^{\circ} 48^{\prime} \mathrm{W}\right)$, alt ca. $1800 \mathrm{~m}$, col. D. M. Vital 7278, 21-VII-1977, det. Lin. (SP 136351); idem, on branches of tree in a space forest, Serra da Bocaina $\left(22^{\circ} 43^{\prime}\right.$ S, $44^{\circ} 42^{\prime}$ W), alt. ca. $1900 \mathrm{~m}$, col. D. M. Vital 7339, 22-VII-1977 (SP 136389); município de Bananal, Nova Suiça, Sítio da Cachoeira, Km. 22 da SP-247, sobre tronco vivo $\pm 2 \mathrm{~m}$ de alt. num barranco úmido perto da cachoeira, col. O. Yano 1231, 26-IX-1978 (SP 147288); idem, sobre tronco vivo na margem do riacho, col. O. Yano 1240, 26-IX-1978 (SP 147294); município de Arujá, Vertentes do Arujá, no tronco vivo, $\pm 1 \mathrm{~m}$ do solo, na mata, col. O. Yano 3459, 21-VI-1981 (SP 170838); Ilhabela, estrada sul, $\pm 20 \mathrm{Km}$ da cidade, sobre pedras grandes junto do rio, col. O. Yano 5158, 31-XII-198 (SP 172575); município de Cananéia, Ilha Cardoso, growing $11 / 2 \mathrm{~m}$ up on a tree trunk in a humid but well illuminated low forest, ca $2 \mathrm{Km}$ from the sea in the northern side of island, col. D. M. Vital 3178, 23-V-1974 (SP 122824); idem, na base onde estão as instalações, crescendo junto com orquídeas no tronco de árvore de mata, col. O. Yano 596, 17-XI-1976 (SP 136656); idem, Poço da Anta, no tronco vivo da mata, col. O. Yano 3906, 3-III-1982 (SP 171806); idem sobre ramos de arbusto, em uma área brejosa, ca. $2 \mathrm{Km} \mathrm{S}$ do alojamento, col. D. M. Vital 170743, I-XI-1982 (SP 172298); idem, $\pm 25^{\circ} 07^{\prime} \mathrm{S}, 47^{\circ} 57^{\prime} \mathrm{W}$, on rocky cliff near top of morro do Cardoso, col. D. M. Vital 11351, 14-III-1984, det. D. M. Vital como $P$. fulgens (SP 190520); município de Cananéia, Ilha Comprida, picada a direita da estrada, sobre tronco vivo, formando tufos pendentes, col. O. Yano \& J. R. Pirani 5994, 25-III-1983 (SP 173083); município de Biritiba Uçu, no Km 74-75 da SP-98, sobre tronco vivo, mata secundária, col. O. Yano, K. C. Pôrto \& J. R. Pirani 7817, 25-VII-1983 (SP 189277); idem, na margem do rio Guaçu, sobre tronco de árvore viva, mata ciliar, col. O. Yano, K. C. Pôrto \& J. R. Pirani 7848, 25-VII-1983 (SP 189306); município de São Sebastião, Rio Boiçucanga, sobre tronco vivo, mata ciliar, col. O. Yano, D. P. da Costa \& J. R. Pirani 7913, 26-VII-1983 (SP 189368); município de Caraguatatuba no Km 77 da estrada SP-99, sobre tronco vivo, margem do riacho encachoeirado, col. O. Yano, K. C. Pôrto \& J. R. Pirani 7975, 28-VII-1983 (SP 189424)? município de Apiaí, 24 $32^{\prime} \mathrm{S}, 48^{\circ} 49^{\prime} \mathrm{W}$, in direct sunlight, on a steep and very humid bank, ca. 4Km SE of Apiaí, col. D. M. Vital 11174, 26-VIII-1983, det. D. Vital como P. fulgens (SP 189934); município Embu Guaçu, sítio Saito no Km 62,5 da SP-242, sobre tronco vivo, mata ciliar, col. O. Yano \& L. Y. Kida8655, 14-XI-1983 (SP 190381); município de Peruíbe, Guarau, Estação Ecológica de Juréia, sobre tronco vivo na restinga, col. col. O. Yano \& Zélia R. de Mello 11707, 9-X-1988 (SP 222981). Paraná, município de São João do Triunfo, pendulous on branches, col. D. M. Vital 3316. 20-VI-1974, det. D. Griffin III (SP 122939); between Curitiba and Joinville on BR-376, Km 51 $\left(25^{\circ} 50^{\prime} \mathrm{S}, 49^{\circ} 05^{\prime} \mathrm{W}\right)$, in cloud forest with thick undergrowth of bamboos and 
scattered very tal Araucaria angustifolia, on tree trunks and rotting, col. Dale Vitt 20830, 31-VIII-1977 (H. U. Alberta, SP 148417); Ponta Grossa, na mata de galeria, epífita, col. L. Krieger 18060, 17-V-1979 (SP 170483); município de Campo do Tenente, sobre casca de árvore junto ao Rio Vermelho no cruzamento com a BR-116, $\pm 25^{\circ} 55^{\prime}$ S, $49^{\circ} 38^{\prime} \mathrm{W}$, col. D. M. Vital 9469, 23-VII-1980 (SP 149321); município de São Mateus do Sul, $\pm 10 \mathrm{Km}$ do sul, sobre tronco de árvore viva, mata secundária, col. O. Yano, J. R. Pirani 6459, 20-IV-1983 (SP 173328); município de Bocaiúva do Sul, Fazenda Pato Branco, sobre galhos de bracatinga, mata úmida, col. O. Yano \& F. C. da Silva 11318, 28-IV-1988 (SP 222260); município de Piraquara, Mananciais da Serra, epífitas, col. Y. S. Kuniyoshi s/n, V-1988 (SP 222921). Santa Catarina, Serra Geral, col. E. Ule, I-1890, det. Mueller (SP 32973); Araranguá, Serra da Pedra, in arbore, col. R. Reitz 873, 28-XII-1943 (ASSL 2882); ilha de Santa Catarina, Morro do Antão, ad arborum il silva, col. A. Sehnem, 22-I-1948 (ASSL 3242, SP 222960); Bom Retiro, Campo dos Padres, in árvore, col. A. Sehnem, 18-I-1957 (ASSL 7094b); Serra Geral, Serra do Rio Rastro, ca. 12Km W of Bom Jardim da Serra, on road to Lauro Mueller, at rim of summit plateau, $1470 \mathrm{~m}$, ca. $28^{\circ} 22^{\prime} \mathrm{S}$, 49 32'W, humid hardwoods, col. D. M. Vital \& W. R. Buck 12352, 12366, 27-IX-1984 (SP 207352; SP 207362; NY). Rio Grande do Sul, São Leopoldo, Sapucaia, in ramulis, col. A. Sehnem, 25-XI-1935, det. T. Herzog como $P$. immersum (ASSL 2875, SP 50828); idem, col. A. Sehnem, 10-IX-1941 (ASSL 324); idem, faz. São Borja, in arbore in silva, col. A. Sehnem, 8-V-1935 (ASSL 68); Montenegro, Campestre, alt. 400m, ad arborum, col. A. Sehnem s/n, 30-IX-1946, det. A. Sehnem como P. immersum (ASSL 2184, SP 192142); idem, Estação São Salvador, in arbore in silva, col. A. Sehnem, 15XII-1935 (ASSL 2874, SP 222959); São Francisco de Paula, Taimbé, alt. $1000 \mathrm{~m}$, ad arborum, col. A. Sehnem s/n, 19-XII-1950, det. A. Sehnem como $P$. immersum (ASSL 5288, SP 192143; ASSL 5340d); idem, col. A. Sehnem, 19-XII-1959 (ASSL 4570; ASSL 4607; SP 222961; ASSL 4643); idem, Faz. Englert, ad arvorum, col. A. Sehnem, 30-XII-1953 (ASSL 6601); idem, ad ramulum in silva nebulari, col. A. Sehnem, 15-II-1956 (ASSL 6826; ASSL 6851; ASSL 6839d); idem, Taimbé, ad truncos arborem in silva, col. A. Sehnem, 16II-1953 (ASSL 6388, SP 222964); idem, Serra do Faxinal, ad arborum, col. A. Sehnem, 18-XII-1950 (ASSL 5308, SP 222963); Bom Jesus, in arbore, col. A. Sehnem, 14-I-1942 (ASSL 573, SP 222956); idem, Serra da Rocinha, ad arbores, col. A. Sehnem, 18-I-1950 (ASSL 4803, SP 222962); idem, ad fl. Tainhas, ad ramos in silva, col, A. Sehnem, 20-II-1952 (ASSL 5996); Aparados da Serra, Serra da Rocinha, ad ramum pedens, col. A. Sehnem, 14-I-1942 (ASSL 213c); idem, col. A. Sehnem, 3-II-1953 (ASSL 6370); município de Cambará do Sul, estrada para Taimbezinho, sobre tronco vivo, mata de Araucaria, col. col. O. Yano \& J. R. Pirani 6992, 26-IV-1983 (SP 182066); idem, Parque Nacional de Aparados da Serra, ca. $29^{\circ} 08^{\prime} \mathrm{S}, 50^{\circ} 05^{\prime} \mathrm{W}$, near Itaimbezinho, Araucaria dominated cloud forest, col. D. M. Vital \& W. R. Buck 12236, 12260 , 12264, 12281, 12282, 12294 e 12306, 26-IX-1984 (SP 207285); Caxias do Sul, ad arborem in silva, col. A. Sehnem, 12-I-1947 (ASSL 2645, SP 222958); 
idem, col. A. Sehnem, 14-I-1947 (ASL 2612a); idem, Vila Oliva, col. A. Sehnem, 16-I-1947 (ASSL 2624, SP 222957); Gramado, ad arborem in silva, col. A Sehnem, 28-XII-1949 (ASSL 4740); Santa Cruz do Sul, Pinheiral, ad ramos arboris in silva, col. A. Sehnem, 22-XII-1952 (ASSL 6186); Vacaria, ad arborem in silva, col. A. Sehnem, 16-I-1952 (ASSL 5958).

\section{Comentários}

Phyllogonium viride Brid. cresce em galhos e troncos de árvores e arbustos de mata Atlântica e mata de Restinga, raramente sobre pedras e barrancos úmidos.

A espécie é reconhecida pelo aspecto pendente, porém é mais vistosa pelo seu tamanho e brilho; filídios em forma navicular ou cimbiforme, ápice agudo, fortemente recurvado e dispostos disticamente; lâminas do filídio de superfície lisa, freqüentemente com estrias longitudinais, quando seco; células estreitas alongadas com paredes tipicamente estranguladas. Quando fértil, cápsula distintamente imersa, estômatos ausentes e calíptra pequena, cuculada e densamente pilosa.

Segundo literatura, $P$. viride ocorre nos Estados de Pernambuco, Bahia, Minas Gerais, Rio de Janeiro, São Paulo, Paraná, Santa Catarina e Rio Grande do Sul. Está sendo referida pela primeira vez para os Estados de Alagoas e Espírito Santo e também novas localidades nos Estados acima mencionados (Fig. 15).

Phyllogonium viride é uma das espécies utilizadas pelas aves para a construção de ninhos, sendo observado nas regiões de restinga.

As quatro espécies que são consideradas sinônimos de $P$. viride por Lin (1983), realmente não apresentam diferenças morfológicas e anatômicas, apenas o tamanho das plantas ou melhor, a largura dos ramos, varia muito. Foi observado que as plantas masculinas são realmente mais estreitas, isto também relatado por Sehnem (1969). Com isto, no Brasil temos um único representante $P$. viride.

\section{Agradecimentos}

As autoras agradecem aos curadores dos Herbários do Instituto Anchietano de Pesquisas e Jardim Botânico do Rio de Janeiro, pelo empréstimo de material.

\section{Referências Bibliográficas}

BUCK, W. R. \& IRELAND, R. R. 1985. A reclassification of the Plagiotheciaceae. Nova Hedwigia, 41: 89-125.

LIN, S.-H. 1983. A taxonomic revision of Phyllogoniaceae (Bryopsida) Part I. J. Taiwan Mus., 36(2): 37-87. 
LIN, S.-H. 1984. Re-classification of Phyllogoniaceae sensu lato. J. Hattori Bot. Lab., 55: 295-301.

SEHNEM, A. 1969. Musgos sul-brasileiros. Pesquisas, 27: 1-36.

YANO, O. 1979. Contribuição ao inventário dos Musci Brasileiros: Helicophyllaceae. Rickia, 8: 7-16.

YANO, O. 1981a. A checklist of Brazilian mosses. J. Hattori Bot. Lab., 50: 279-456.

YANO, O. 1981b. Contribuição ao inventário dos musci brasileiros: 2. Phyllodrepaniaceae. Acta Amazonica, 11(3): 505-509.

YANO, O. 1981c. Aytoniaceae (Marchantiales, Hepaticopsida) no Brasil. Revta. brasil. Bot., 4(2): 89-94.

YANO, O. 1984a. Novas ocorrências de Helicophyllum torquatum (Hook.) Brid. (Helicophylaceae, Bryopsida) no Brasil. Rickia, 11: 35-42.

YANO, O. 1984b. Contribuição ao inventário dos musci brasileiros: 3. Racopiaceae (Bryopsida, Isobryales). Revta. brasil. Bot., 7(1):57-63.

YANO, O. 1984c. Briófitas. In Fidalgo, O. \& Bononi, V. L. R., coord. Técnicas de coleta, preservação e herborização de material botânico. São Paulo, Instituto de Botanica. 62p. (Manual no 4).

YANO, O. 1985. Contribuição ao inventário dos Musci Brasileiros: 4. Rhachitheciaceae. Rickia, 12: 29-34.

YANO, O. 1986. Contribuição ao inventário dos Musci Brasileiros: 5. Rhizogoniaceae (Bryopsida). Rickia, 13: 49-60.

YANO, O. \& ANDRADE-LIMA, D. 1987. Briófitas do Nordeste Brasileiro: Estado de Pernambuco. Revta. brasil. Bot., 10: 171-181. 


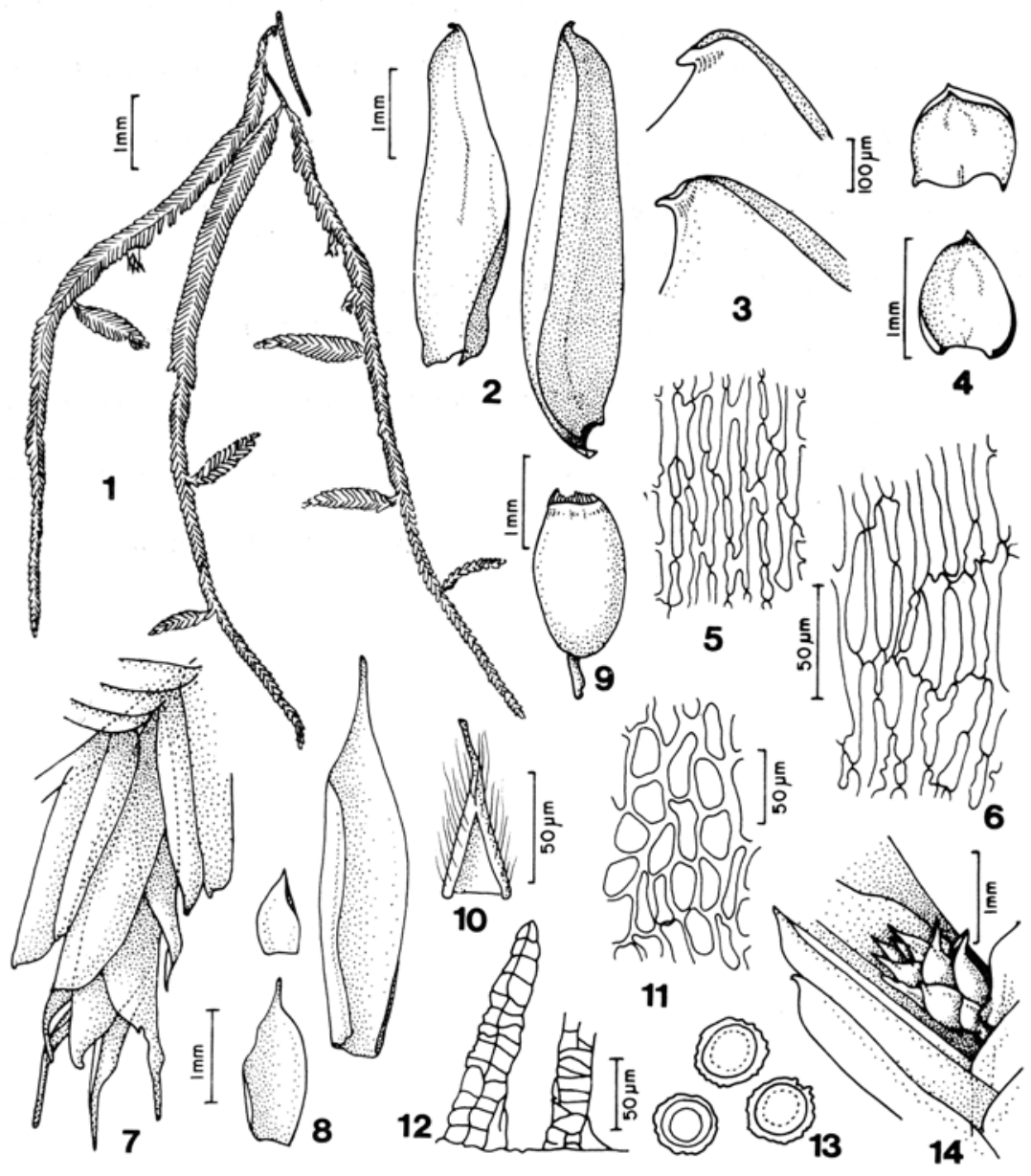

Figuras 1-14. Phyllogonium viride.

1. Aspecto geral do gametófito. 2. Filídios do ramo pendente. 3. Ápice do filídio. 4. Filídios do ramo prostrado. 5. Células da região mediana do filídio. 6. Células da base do filídio. 7. Esporófito. 8. Filídios periqueciais. 9. Cápsula. 10. Calíptra :ılosa. 11. Células da parede da cápsula. 12. Dentes peristomiais. 13. Esporos. 14. Anteridióforo. 
Estudos de Briófitas do Brasil...

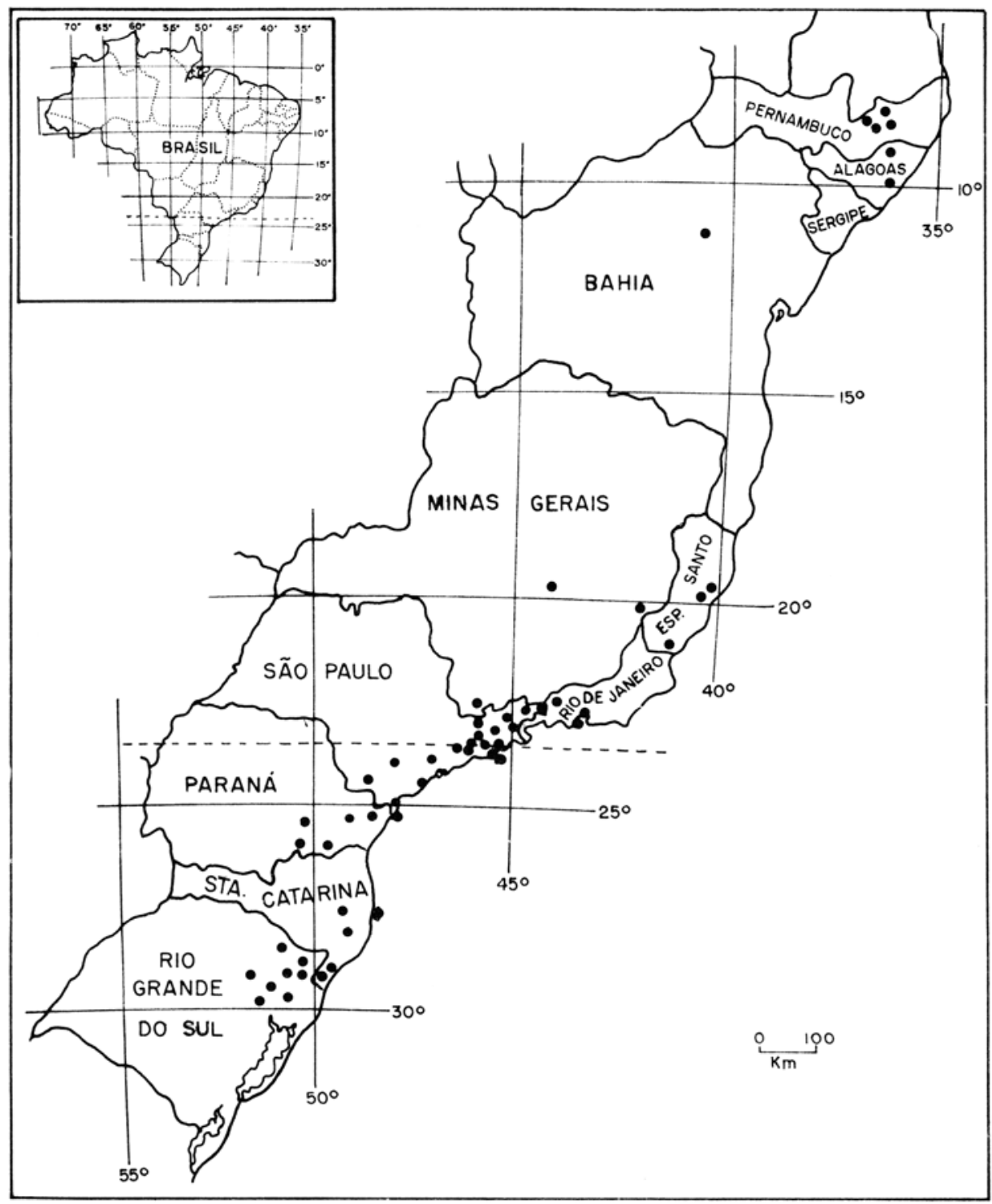

Figura 15. Distribuição geográfica brasileira de Phyllogonium viride. 